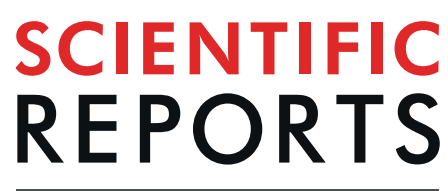

natureresearch

\title{
Silicon confers protective effect against ginseng root rot by regulating sugar efflux into apoplast
}

\author{
Ragavendran Abbai ${ }^{1}{ }^{1}$, Yu-Jin Kim ${ }^{2}$, Padmanaban Mohanan ${ }^{1}$, Mohamed El-Agamy Farh ${ }^{1}$, \\ Ramya Mathiyalagan ${ }^{1}$, Dong-Uk Yang ${ }^{2}$, Suriyaprabha Rangaraj ${ }^{3}$, Rajendran Venkatachalam ${ }^{3,4}$, \\ Yeon-Ju Kim ${ }^{2 *} \&$ Deok-Chun Yang $\mathbb{D}^{1,2^{*}}$
}

Root rot caused by llyonectria mors-panacis is a devastating fungal disease leading to defect in root quality and causes reduced yield during the perennial life cycle of Panax ginseng Meyer. This indicates the imperative need to understand the molecular basis of disease development and also to enhance tolerance against the fungus. With this idea, the protective effect of silicon (supplied as silica nanoparticles) in $P$. ginseng root rot pathosystem and its molecular mechanism was investigated in the current study. We have tested different concentrations of silicon (Si) to disease-infected ginseng and found that long term analysis ( $30 \mathrm{dpi}$ ) displayed a striking $50 \%$ reduction in disease severity index upon the treatment of Si. Expectedly, Si had no direct degradative effect against the pathogen. Instead, in infected roots it resulted in reduced expression of PgSWEET leading to regulated sugar efflux into apoplast and enhanced tolerance against $I$. mors-panacis. In addition, under diseased condition, both protopanaxadiol (PPD) and protopanaxatriol (PPT) type ginsenoside profile in roots were higher in Si treated plants. This is the first report indicating the protective role of $\mathrm{Si}$ in ginseng-root rot pathosystem, thereby uncovering novel features of ginseng mineral physiology and at the same time, enabling its usage to overcome root rot.

Panax ginseng Meyer is an Oriental medicinal adaptogen and ginsenosides are the major pharmacologically active components of ginseng, which is proved to be effective against various diseases ${ }^{1}$. It is perennial in nature and the transition from vegetative to reproductive phase occurs at the third year and the accumulation of ginsenosides in roots increases with age ${ }^{2}$. Ginseng root rot caused by the fungus, Ilyonectria mors-panacis is one of the devastating diseases which initially infects the root tip and then proceeds until the crown. In addition, replanting results in infection of new plants. Hence, there is an imperative need to design strategies to overcome ginseng root rot. Younger age ( 2 years) of the plant, acidic soil ( $\mathrm{pH} 5.5-6.0)$, soil temperature $\left(18-20^{\circ} \mathrm{C}\right)$, high iron content are the major factors that promote the occurrence of root rot $^{3,4}$.

Pathogen invades a plant to acquire nutrients which are majorly sugars, to support their growth and replication $^{5}$. Understanding the molecular signaling events during plant-pathogen interaction is of great importance to establish strategies to overcome the pathogen. The plant defense system initially detects the pathogen, followed by the activation of the appropriate signal cascades. The downstream defense responses especially the crucial role of hormonal pathways such as SA (Salicylic acid), JA (Jasmonic acid) and Ethylene (ET) mediated pathways are well established. Periodic global transcriptome analyses by RNAseq revealed that JA and ET are majorly activated in ginseng-root rot pathosystem ${ }^{6}$. Previously, JA had been demonstrated to influence the triterpenoid pathway in ginseng and PgSE2 was found to influence phytosterol biosynthesis ${ }^{7,8}$. Phytosterols are essential component of the plasma membrane that determines its rigidity/fluidity. Certain pathogens have the ability to modify the composition of the phytosterols in the plasma membrane to alter nutrient efflux ${ }^{9,10}$.

\footnotetext{
${ }^{1}$ Graduate School of Biotechnology, College of Life Science, Kyung Hee University, Yongin, 446-701, South Korea. ${ }^{2}$ Department of Oriental Medicinal Biotechnology, College of Life Science, Kyung Hee University, Yongin, 446-701, South Korea. ${ }^{3}$ Centre for Nanoscience and Technology, K. S. Rangasamy College of Technology, Tiruchengode, 637215, Tamil Nadu, India. ${ }^{2}$ Dr. N.G.P Arts and Science College, Kalpatti road, Coimbatore, 641048, Tamil Nadu, India. *email: yeonjukim@khu.ac.kr; dcyang@khu.ac.kr
} 
However, the molecular basis of root rot development in ginseng remains elusive and has to be addressed to enhance tolerance against the fungal disease. Perennial life cycle of ginseng, lack of allelic variations and difficulty in regeneration makes genetic improvement for root rot tolerance challenging at the moment. Therefore, priming of defense responses is considered as one of the feasible options. In a recent study, Bacillus amyloliquefaciens AK-0 was found to be antagonistic to Cylindrocarpon destructans and decreased the disease severity index in 4-year-old ginseng ${ }^{11}$. However, its interaction with other rhizobacteria and its effect on younger aged ginseng (where root rot occurrence is predominant) is unknown. Considering the present scenario, the application of 'protective agents' that specifically induce plant defense responses upon pathogen invasion seems to be one of the promising strategies to overcome root rot.

The protective role of silicon in Arabidopsis-powdery mildew pathosystem is well documented. It has been confirmed to induce defense related genes and at the same time balance primary metabolism ${ }^{12}$. Similarly, over the years its stress responsive role has been demonstrated in many other scenarios as well ${ }^{13-16}$. Of late, Phytonanotechnology i.e. the application of nanoparticles for empowering agriculture, is of great interest. The uptake and translocation of mesoporous silica nanoparticles in maize is already demonstrated ${ }^{17,18}$. In addition, silica nanoparticles were found to be compatible in A. thaliana up to $1000 \mathrm{mg}$. $\mathrm{L}^{-1} 19$.

Based on this background, it was hypothesized that "Si might have protective effect in ginseng-root rot pathosystem when supplied as silica nanoparticle". A series of experiments involving phenotyping in the presence and absence of $\mathrm{Si}$, expression profiling of potential candidate genes/metabolites was carried out to unravel the role $\mathrm{Si}$ against ginseng root rot and its molecular basis. Overall, this study provides novel insights into the molecular factors governing defense responsiveness of $\mathrm{Si}$ and also on mineral physiology in the ginseng root-rot pathosystem.

\section{Results}

The protective role of Si in $\mathrm{P}$. ginseng-root rot pathosystem. Various treatments include mock (HC), non-infected $+1 \mathrm{mM} \mathrm{Si}(\mathrm{H}-\mathrm{Si} 1 \mathrm{mM})$, non-infected $+2 \mathrm{mM} \mathrm{Si}(\mathrm{H}-\mathrm{Si} 2 \mathrm{mM})$, infected without silicon supplementation (IS), infected $+1 \mathrm{mM} \mathrm{Si}$ (I-SilmM) and infected $+2 \mathrm{mM} \mathrm{Si}(\mathrm{I}-\mathrm{Si} 2 \mathrm{mM})$.

At prolonged phase II (30 dpi), the seedlings were categorized into 6 grades based on the root phenotype and the disease severity index (DSI) was determined (Table S1; Fig. 1a). As expected, upon $1 \mathrm{mM}$ and $2 \mathrm{mM}$ administration of Si a striking 50\% reduction in DSI was recorded, which confirms its protective effect against ginseng-root rot (Fig. 1b-d).

In addition, the shoot and root biomass were significantly improved in both I-Sil mM and I-Si2mM, than the IS (Fig. 1e). Furthermore, upon Si administration under infected conditions the total major ginsenosides in the root i.e., both PPD type $(\mathrm{Rb} 1+\mathrm{Rc}+\mathrm{Rb} 2+\mathrm{Rd})$ and PPT type $(\mathrm{Rg} 1+\mathrm{Re}+\mathrm{Rf})$ were considerably improved in comparison with IS (Fig. 1g). However, in the absence of pathogen, Si treatment did not have any significant effect on the phenotype.

Silica nanoparticles possesses no anti-fungal activity against $I$. mors-panacis. In-vitro screening was performed to examine the direct anti-fungal activity of silica nanoparticles against I. mors-panacis. Expectedly, it was found that the growth rate of the fungal pathogen was undisturbed even at $4 \mathrm{mM}$ concentration (Figs. $1 \mathrm{f}$ and S1). This confirms that it does not have any direct anti-fungal activity which is consistent with previous reports in other plant species.

Si regulates genes involved in Jasmonic acid (JA) signaling, triterpene, phytosterol biosynthesis and sugar efflux. The phenotype of the seedlings at early phase ( $4 \mathrm{dpi})$, intermediate phase $(8 \mathrm{dpi})$ and prolonged phase I ( $16 \mathrm{dpi}$ ) were observed prior to gene expression studies. Considerable improvement in terms of root rot tolerance was evident in each phase when $\mathrm{Si}$ was treated. Until the intermediate phase, I-SilmM was almost similar to the non-infected seedlings with respect to shoot and root morphology (Fig. 2a,b). In addition, the phenotype of I-SilmM in prolonged phase I was noticeably better than the IS (Fig. 2c).

Expression profiling of potential candidate genes was carried out using qRT-PCR. The expression of commonly used house-keeping genes such as Pgactin, PgGAPDH \& PgCYP were evaluated and PgGAPDH was found to be ideal among them, hence was used as endogenous reference for profiling the target genes (Fig. S2). To understand the Si uptake into ginseng roots, silicon influx transporter, Pglsil was identified from ginseng EST library based on homology analysis with orthologs of model plants. The expression of Pglsil was induced in I-SilmM, whereas it was significantly suppressed in IS (Fig. S3a). Under non-infected condition, when Si was treated, the expression of silicon influx transporter was unaltered.

To further determine the candidate pathways associated with biotic tolerance mediated by $\mathrm{Si}$, the expression profile of major genes regulating JA biosynthesis and signaling, triterpene and phytosterol biosynthesis and sugar efflux pathways were analyzed. Further, the associated metabolites were also quantified. Considering that JA is one of key signaling molecule for defense response and also ginsenoside accumulation, JA biosynthesis and signaling related genes were investigated to trace out the molecular framework of Si mediated defense responses against the fungus. Initially, JA biosynthesis associated gene, PgLOX6 (Fig. S3b) was profiled, and then the expression of JA signaling repressor, $P g T I F Y 10 A$ (Fig. S3c) was determined and finally Methyl Jasmonate was quantified (Figs. S4 and S5). These suggested that Si induced JA biosynthesis and signaling in response to I. mors-panacis infestation.

Further, the probable potential candidate transcription factors (TFs) involved in JA mediated activation of ginsenoside biosynthesis such as $P g M Y C 2 a, P g M Y C 2 b, P g W R K Y 22$ and $P g M Y B 3$ were profiled. Interestingly, in I-SilmM these TFs expression were found to be induced in stage specific manner. $P g M Y C 2 b$ was induced in early phase, $P g M Y B 3 \& P g W R K Y 22$ up-regulated in the intermediate phase, whereas $P g M Y C 2 a$ was enhanced in prolonged phase I (Fig. S3d-g, Table S2). On the other hand, all the above TFs were suppressed in IS. 
(a)

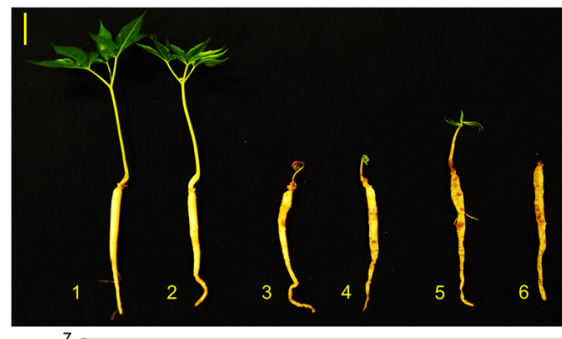

(c)

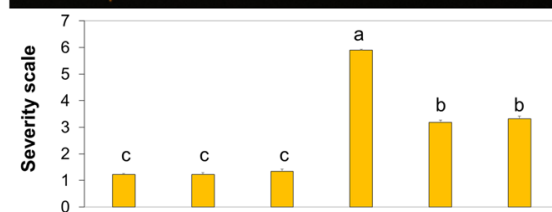

(e)

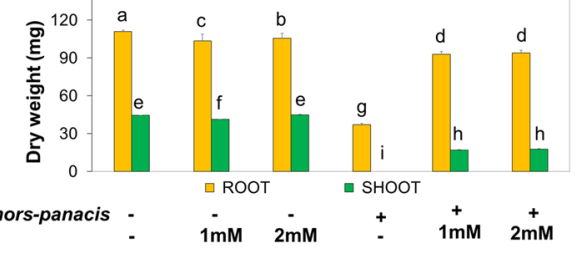

(b)

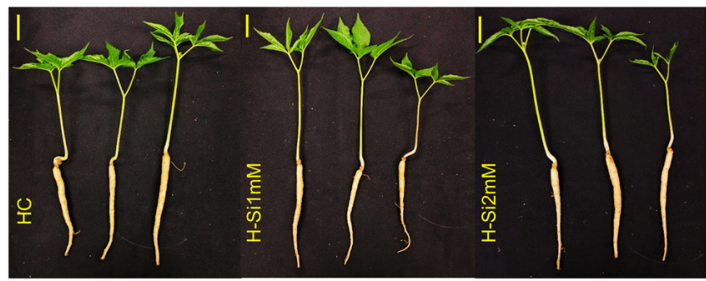

(d)

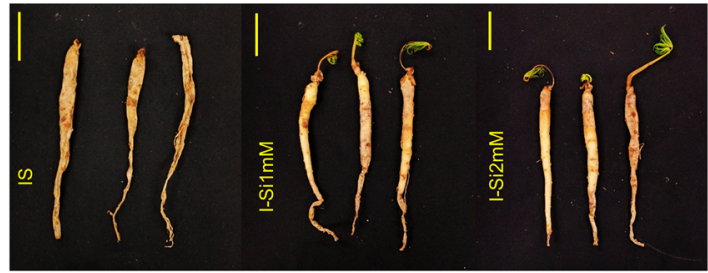

(f)

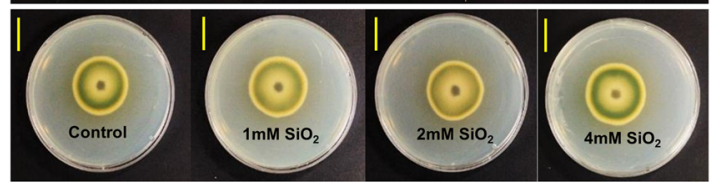

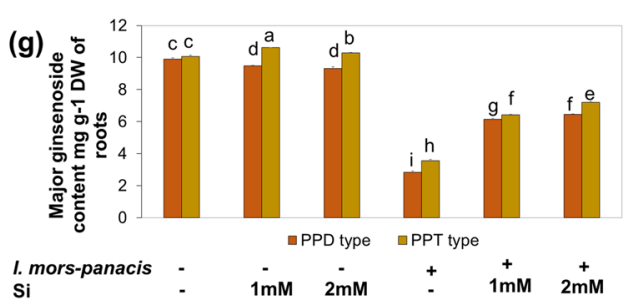

Figure 1. The protective effect of Si at Prolonged phase II. (a) Grading of plants into six categories on the basis of infection level in roots. (b) Non-infected seedlings did not show significant difference in phenotype due to Si supplementation. (c,d) Striking 50\% reduction of disease severity index (DSI) in the Si treated seedlings was observed. No significant difference between the performance of $1 \mathrm{mM}$ and $2 \mathrm{mM}$ treatment of Si under infected conditions. (e) Si treatment reflected positive impact on Root and shoot biomass under pathogen infestation. (f) Silica nanoparticles did not display any direct degradative effect against $I$. mors-panacis. (g) Drastic decrease in total major ginsenoside profile was observed under infected conditions and interestingly a significant increase was documented in both PPD and PPT type of ginsenosides upon Si administration under infected conditions. Note: Scale: $2 \mathrm{~cm}$; mock $(\mathrm{HC})$, non-infected $+1 \mathrm{mM} \mathrm{Si}(\mathrm{H}-\mathrm{Si} 1 \mathrm{mM})$, non-infected $+2 \mathrm{mM} \mathrm{Si}$ (H-Si2mM), infected without silicon supplementation (IS), infected $+1 \mathrm{mM} \mathrm{Si}$ (I-SilmM) and infected $+2 \mathrm{mM}$ $\mathrm{Si}(\mathrm{I}-\mathrm{Si} 2 \mathrm{mM})$; PPD type: Rb1 + Rc + Rb2 + Rd; PPT type: Rg1 + Re + Rf.

Regulation of mevalonic acid pathway to induce phytosterol accumulation. Expression profile of the rate-limiting genes of the mevalonic acid pathway, namely, PgHMGR1, PgHMGR2 and PgSS1 (Fig. S3h-j) clearly indicated the activation of Mevalonic acid pathway in I-SilmM. However, the expression of genes associated with ginsenoside pathway such as PgSE1 \& PgDDS (Fig. S3k,l) was unaltered, while PgBAS (Fig. S3m) was down-regulated in I-SilmM. Conversely, in the IS treatment, all the above mentioned genes were significantly down-regulated.

Next, the expression of key genes involved in the phytosterol biosynthesis such as PgSE2, PgCAS and PgLAS (Fig. 3a-c) was determined. Also, obtusifoliol $14 \alpha$-demethylase, $\operatorname{PgCYP51}$ (Fig. S3n) was identified from ginseng EST library by homology search. The expression profile of these genes indicated the induction of sterol biosynthesis in I-SilmM, whereas, it was severely down-regulated in the IS. Furthermore, the metabolite profile of $\beta$-sitosterol and stigmasterol was in line with the observed transcript abundance, thus confirming sterol accumulation in I-SilmM and at the same indicating low sterol biosynthesis in IS (Figs. 3e and S4).

Regulated sugar efflux into apoplast. Finally, the focus was on analyzing the sugar efflux into apoplast. Hence, sugar efflux transporter, SWEET (Sugars Will Eventually be Exported Transporters), which is important for sugar efflux into apoplast was identified in P. ginseng from the previously available EST library. The expression of PgSWEET was suppressed to a great extent during P. ginseng-I. mors-panacis interaction in the presence of $\mathrm{Si}$ (in I-SilmM), which indicates the possibility regulated sugar efflux into apoplast. Contrastingly, in the IS, PgSWEET was significantly induced which sheds light on enhanced sugar efflux into apoplast (Fig. 3d).

Furthermore, the total and reducing sugars were profiled in root apoplastic fluid by anthrone and dinitrosalicalic acid DNS methods respectively. Expectedly, in I-SilmM, the sugar content was almost similar to the non-infected samples, while, the sugar profile was significantly high in the IS (Fig. 3g,h). This clearly confirms that $\mathrm{Si}$ strictly regulates the sugar efflux into apoplast in ginseng-root rot pathosystem and on the other hand, the sugar efflux into apoplast is triggered in IS. 
(a)

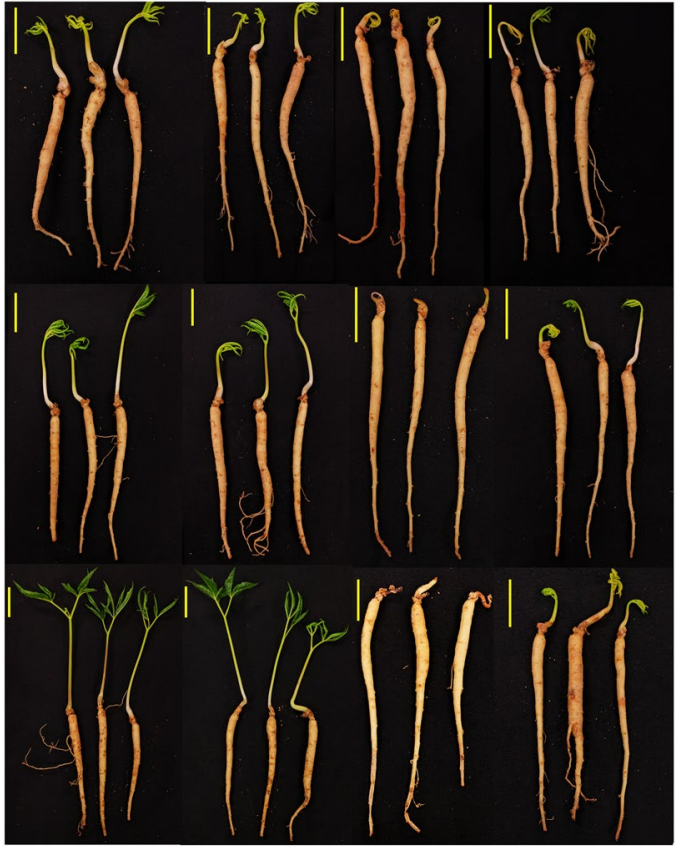

I. mors-panacis

Si

Figure 2. Time-dependent phenotypic analysis reveals the positive role of $\mathrm{Si}$ in enhancing the tolerance ginseng against the fungal pathogen. (a) At the early phase (4 dpi) the Si treated infected seedlings were completely similar to the mock. (b) During the intermediate phase ( $8 \mathrm{dpi})$ rotting initiated at the root tips of the infected seedling in the absence of $\mathrm{Si}$, whereas, the $\mathrm{Si}$ supplemented seedlings were almost similar to the non-infected seedlings. (c) In the Prolonged phase I (16 dpi) severe infestation was observed in the absence of Si and on the other hand in the presence of Si only mild rotting symptoms were noted. Note: Scale: $2 \mathrm{~cm}$; dpi: days post infection.

Si regulates uptake of certain essential minerals. The quantity of candidate minerals such as Iron (Fe), Calcium $(\mathrm{Ca})$, Copper $(\mathrm{Cu})$ and $\mathrm{Zinc}(\mathrm{Zn})$ were estimated in the experimental roots. The Ca content remained largely unaltered across all the treatments and time points, except in the IS at prolonged phase II, wherein Ca was significantly higher than all other treatments. Similarly, Fe, Cu and $\mathrm{Zn}$ profile of the IS at prolonged phase II was also significantly higher than any other treatments (Fig. 4). This suggests that there is a clear variation in the mineral uptake pattern between the I-SilmM and the IS.

\section{Discussion}

The protective role of silicon is well documented across several plant pathosystems ${ }^{13-16,20}$. Silicon is absorbed as silicic acid $\left[\mathrm{Si}(\mathrm{OH})_{4}\right]$ by the plant system and silicon influx transporter, Lsil belongs to an aquaporin membrane protein family (Nod26-like major intrinsic protein (NIP) III which constitutively expresses in roots and coordinates the passive influx of silicic acid $\left[\mathrm{Si}(\mathrm{OH})_{4}\right]^{21,22}$. But the mechanism of silica nanoparticles uptake into plants is not yet established. In the present study, $l s i 1$ was identified in ginseng and its up-regulation suggests that probably silica nanoparticles is somehow converted to silicic acid and then absorbed by the ginseng roots under infected condition. However, further confirmatory studies are essential to unravel the enigma associated with the uptake of Si into ginseng roots.

The total major ginsenoside content in roots was considerably higher in I-SilmM at prolonged phase II as compared to IS. This indicates that ginsenosides might be the key players in overcoming the pathogen. Moreover, the plausible role of ginsenosides as defensive agents has been reported earlier ${ }^{23,24}$. Further, time course dependent global transcriptomic study revealed that majorly JA and ethylene (ET) mediated signaling is activated in ginseng-root rot pathosystem ${ }^{6}$. In another study, $P g L O X 6$ was characterized to be the precursor of JA biosynthesis in ginseng and $P g M Y C, P g W R K Y 22$ were indicated as the probable potential candidate TFs involved in JA mediated ginsenoside biosynthesis ${ }^{7}$. Besides, $P g M Y B 3$ was found to be a JA responsive $\mathrm{TF}^{25}$. In the current study, induction of PgLOX6, suppression of PgTIFY10A in I-SilmM revealed the activation of JA biosynthesis and signaling. Metabolite profile of MeJA re-confirmed the above mentioned. Expression profile of the candidate JA signaling associated TFs indicated that $P g M Y C 2 b$ might be responsive in early phase, $P g M Y B 3$ and $P g W R K Y 22$ might be associated with intermediate phase and $P g M Y C 2 a$ might play a role in prolonged phase I.

In I-SilmM, key upstream genes of Mevalonic acid pathway namely, PgHMGR1, PgHMGR2 \& PgSS1 were upregulated. However, contradicting to our initial hypothesis, Si did not trigger the expression PgSE1, PgDDS $\& P g B A S$ and the ginsenoside biosynthesis cassette was not induced in response to I. mors-panacis infestation. Two squalene epoxide genes namely, PgSE1 and PgSE2, former associated with ginsenoside biosynthesis and the 
(a)

(c)

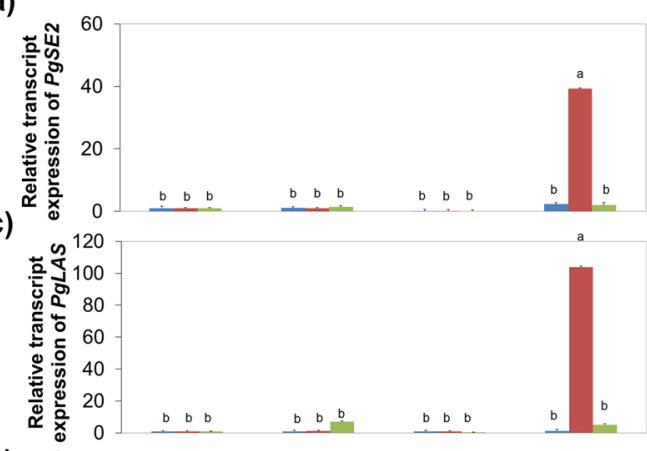

(e)

(g)

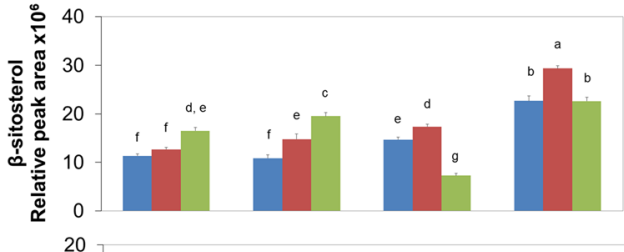

(b)

(d)

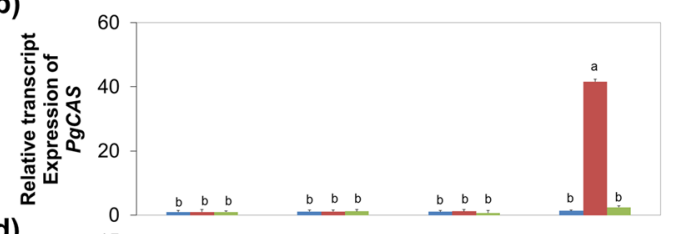

(h)
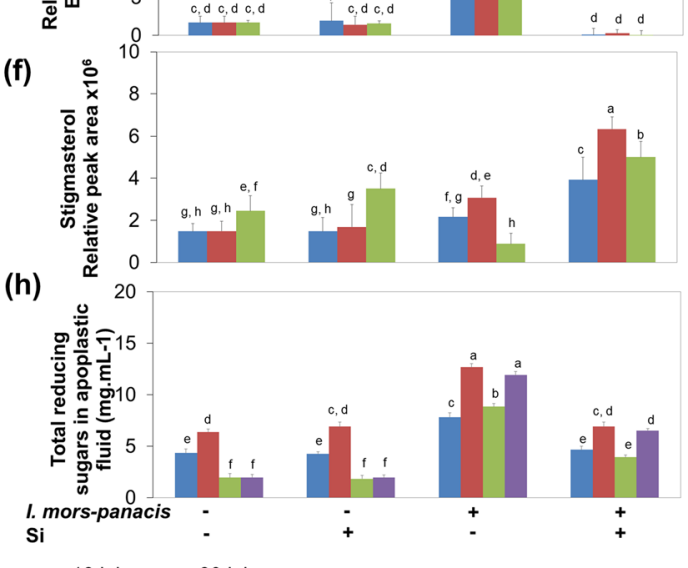

Figure 3. Si regulates sugar efflux into apoplast by increasing membrane sterols. Under infected condition, $\mathrm{Si}$ induces transcriptional re-programming leading to increased expression of (a) PgSE2, (b) PgCAS, (c) PgLAS and (d) reduced expression of the sugar efflux transporter, PgSWEET. Increased levels of (e) $\beta$-sitosterol and (f) stigmasterol confirms sterol accumulation and reduced profile of $(\mathbf{g})$ total sugars, $(\mathbf{h})$ total reducing sugars in the apoplastic fluid indicates regulated sugar efflux into apoplast upon Si treatment under infected conditions.

(a)

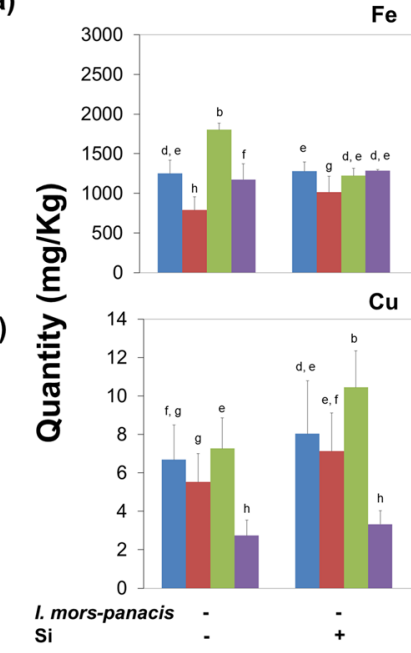

$\mathrm{Fe}$
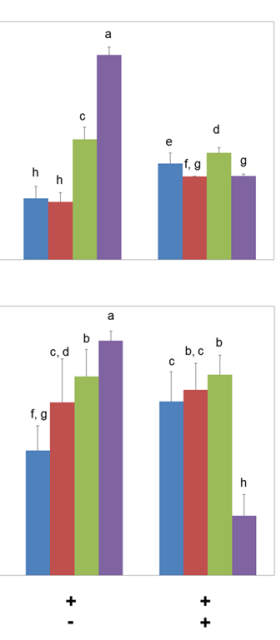

(b)

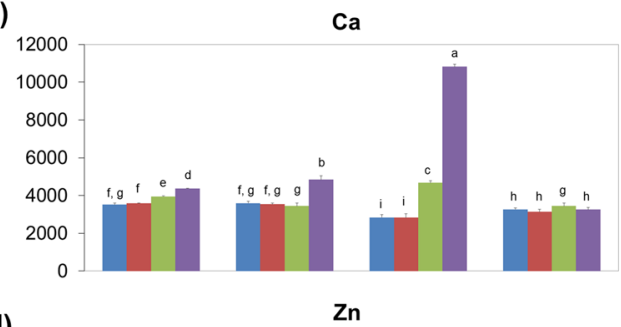

(d)

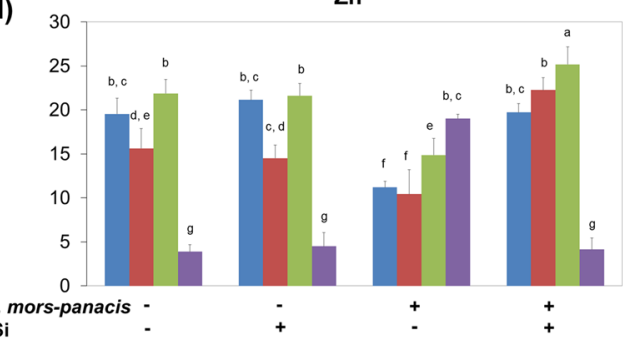

Figure 4. Si influences the uptake of candidate minerals in ginseng-root rot pathosystem. At prolonged phase II (30dpi) increased (a) $\mathrm{Fe}$, (b) $\mathrm{Ca}$, (c) $\mathrm{Cu}$ and (d) $\mathrm{Zn}$ profile was observed under infected condition when $\mathrm{Si}$ were not applied. On the other hand, upon Si administration during infected condition, the quantity of target minerals was similar to the mock.

later involved in the regulation of phytosterols production have been characterized in ginseng ${ }^{8,26}$. Therefore, PgSE2 and other sterol biosynthesis associated genes such as PgCAS, PgLAS, PgCYP51 were insevestigated and they were found to be significantly up-regulated in I-Sil mM. Further, the metabolic profile of $\beta$-sitosterol and 


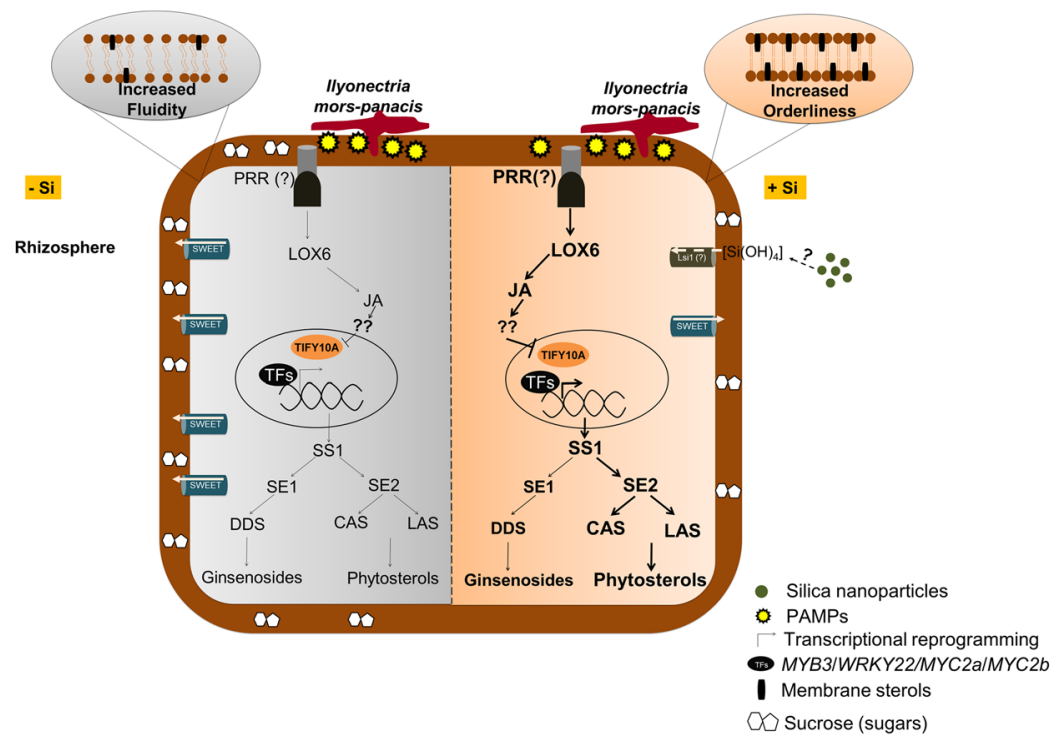

Figure 5. Transcriptional reprogramming mediated by Si leading to enhanced tolerance against I. morspanacis. In the absence of Si higher expression of PgSWEET and reduced phytosterol profile mediated increased sugar efflux into apoplast, thus resulting in severe root rot. On the other hand, Si administration induced transcriptional reprogramming in ginseng roots, leading to regulated sugar efflux into apoplast via JA mediated sterol accumulation, thus enhanced tolerance against I. mors-panacis. Note: Jasmonic acid (JA); Transcription factors (TFs); Pattern Recognition Receptor (PRR).

stigmasterol confirmed that Si orchestrated sterol accumulation under Si supplmentation in root-rot pathosystem. Phytosterols, especially $\beta$-sitosterol, stigmasterol (membrane sterols) play a major role in regulating the orderliness of plasma membrane. Certain PAMPs (Pathogen Associated Molecular Patterns) like cryptogein have the capacity to specifically remove sterols from cell membrane thereby increasing its fluidity and thus resulting in easier nutrient acquisition ${ }^{9}$. In addition, the primary purpose of pathogen invading a plant is reported to be for obtaining nutrients, mostly sugars. Certain pathogenic fungi have the ability to manipulate the membrane bound sugar efflux transporter, SWEET to result in increased sugar efflux into apoplast, thereby supporting its growth and replication ${ }^{27}$. Recently, TAL effectors from Xanthomonas citri was reported to manipulate GhSWEET10, leading to bacterial blight in cotton ${ }^{28}$. Moreover, when stigmasterol is high, the nutrient efflux into apoplast was reported to be significantly reduced leading to tolerance against bacterial pathogens ${ }^{10}$. Similarly, in the current study PgSWEET and the sugar content in the apoplast were profiled to unravel the sugar efflux into apoplast. It was conclusive that sugar efflux into apoplast in I-Sil mM roots was strictly regulated and is the major factor contributing to the tolerance against root rot causing, I. mors-panacis.

Abundance of certain minerals during ginseng cultivation promotes the occurrence of root $\operatorname{rot}^{4}$. Foliar application of iron $(\mathrm{Fe})$ has been correlated with the higher root rot occurrence in Panax quinquefolius. It was further demonstrated that Fe supports growth and sporulation of the fungus. At the infection site, the production of phenolic compounds increases the Fe levels, which is sequestered by the fungus, thereby promoting the occurrence of root $\operatorname{rot}^{29}$. In the present study, interestingly, mineral uptake pattern (Fe, $\left.\mathrm{Ca}, \mathrm{Zn} \mathrm{\&} \mathrm{Cu}\right)$ in I-SilmM was distinct from other treatments in early phase and intermediate phase, while, in the prolonged phase II the IS had a unique pattern of mineral uptake (Fig. S6). In regard with Fe, the IS had the highest quantity, thus adding on to its susceptibility, whereas its profile in I-SilmM roots was similar to that of the non-infected roots, thereby increasing the tolerance towards the pathogen.

Taken together, in IS the reduced phytosterols biosynthesis along with higher expression of PgSWEET enhanced the sugar efflux into apoplast, thereby supporting the growth and replication of the pathogen, resulting in severe root rot. Contrastingly, Si administration induced transcriptional reprogramming in ginseng roots, leading to regulated sugar efflux into apoplast via JA mediated sterol accumulation, thus enhanced tolerance against I. mors-panacis (Fig. 5). Furthermore, this is first study that comprehensively sheds light on the role of $\mathrm{Si}$ in modulating phytosterols accumulation and sugar efflux into apoplast under I. mors-panacis infection and at the same time reveals new insights into ginseng mineral physiology in root rot pathosystem.

\section{Conclusion}

The current study demonstrates the protective effect of Si against ginseng root rot. Interestingly, the root quality and major ginsenoside profile of I-SilmM was much higher than the IS, hinting a positive impact in the market value. Expression and metabolite profiling indicated that Si regulated sugar efflux into apoplast via JA mediated phytostreol accumulation, thus leading to increased tolerance against $I$. mors-panacis. Additionally, the potential candidate root rot responsive genes determined in this study could be employed in molecular breeding programme and also in generating genetically engineered/edited lines to develop novel root rot tolerant ginseng varieties in the future (Fig. 6). Most importantly, Si treatment has the potential to address replant failure. In addition, 


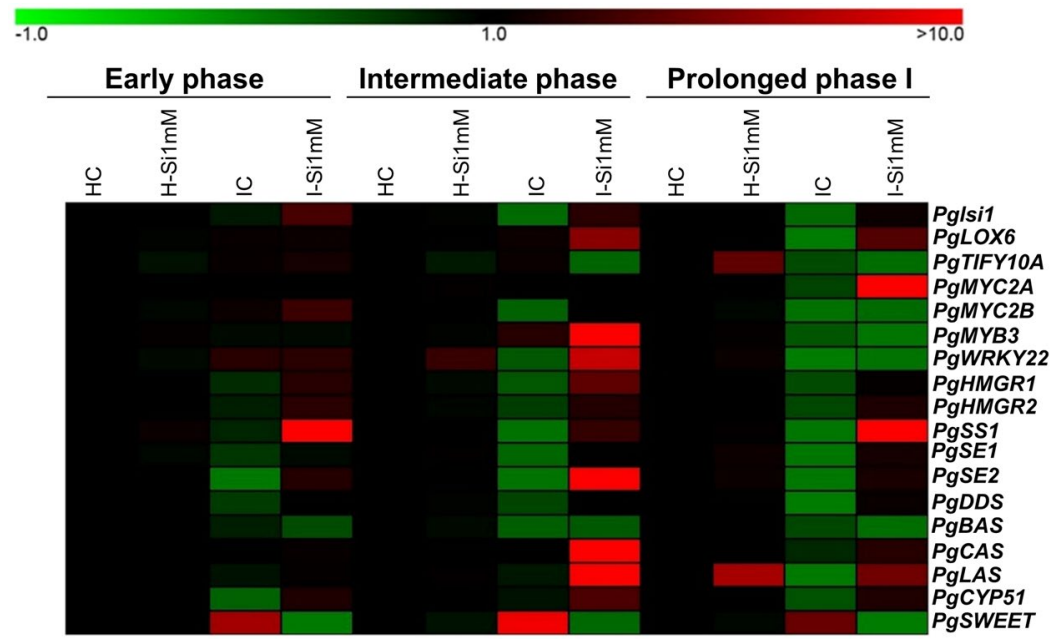

Figure 6. Heat map comprising of all the Si mediated ginseng root rot responsive genes determined in this study. These genes are believed to serve as potential candidates for genetic improvement of ginseng against root rot in the future.

the concentration of the pathogen used in this study is about 90 folds higher than the natural condition, which implies that Si treatment might have more pronounced effect in actual field. Taken together, the current study has open up the avenue for cultivating ginseng with enhanced tolerance against root rot.

\section{Materials and Methods}

Synthesis and characterization of silica nanoparticles. Silica nanoparticles were synthesized from rice husk using acid precipitation method followed by alkali extraction ${ }^{17}$. Rice husk was burnt at $750{ }^{\circ} \mathrm{C}$ using high temperature tubular muffle furnace for $3 \mathrm{~h}$. The obtained rice husk ash (RHA) was treated with $6 \mathrm{~N}$ hydrochloric acid $(\mathrm{HCl})$ under stirring conditions at $70^{\circ} \mathrm{C}$ for $30 \mathrm{~min}$ to leach out impurities under acidic conditions. Then, the ash was washed with distilled water thrice to attain the $\mathrm{pH}$ of 7.0 and $2.5 \mathrm{~N}$ sodium hydroxide $(\mathrm{NaOH})$ solution was added under stirring conditions for $2 \mathrm{~h}$ at $80^{\circ} \mathrm{C}$ to extract silicate from ash. Concentrated sulphuric acid $\left(\mathrm{H}_{2} \mathrm{SO}_{4}\right)$ was gradually added drop wise to the supernatant containing the extracted sodium silicate $\left(\mathrm{Na}_{2} \mathrm{SiO}_{3}\right)$ until reaching $\mathrm{pH} 2.5$, where the solution becomes transparent white silica sol. Then, it was thoroughly washed with double distilled water to remove the sodium interference and consequently washed with ethanol. Finally, the silica powder ( $99.7 \%$ purity) was collected after calcination at $450^{\circ} \mathrm{C}$ for $2 \mathrm{~h}$. The silica nanoparticle size ranged from $20-40 \mathrm{~nm}$ with spherical morphology ${ }^{17}$. The chemical reactions which occurred during the extraction of silica nanoparticles is as follows,

$$
\begin{gathered}
\text { Rice husk ash }(\mathrm{RHA})+(6 \mathrm{~N}) \mathrm{HCl} \stackrel{\text { Leaching }}{\longrightarrow} \mathrm{RHA}+\text { Metal impurities } \\
\mathrm{RHA}+(2.5 \mathrm{~N}) \mathrm{NaOH} \stackrel{\text { Alkali extraction }}{\longrightarrow} \mathrm{Na}_{2} \mathrm{SiO}_{3}+\mathrm{H}_{2} \mathrm{O} \\
\mathrm{Na}_{2} \mathrm{SiO}_{3}+\mathrm{Con} . \mathrm{H}_{2} \mathrm{SO}_{4} \stackrel{\text { Acid precipitation }}{\longrightarrow} \mathrm{Si}(\mathrm{OH})_{4}+\mathrm{Na}_{2} \mathrm{SO}_{4} \\
\mathrm{Si}(\mathrm{OH})_{4}(\text { Wet }) \stackrel{\text { Drying at } 100^{\circ} \mathrm{C}}{\longrightarrow} \mathrm{Si}(\mathrm{OH})_{4}(\text { Dry }) \\
\mathrm{Si}(\mathrm{OH})_{4} \text { (Dry) } \stackrel{\text { Calcination at } 450^{\circ} \mathrm{C}}{\longrightarrow} \mathrm{SiO}_{2}+\mathrm{H}_{2} \mathrm{O}
\end{gathered}
$$

Plant materials, infection and growth parameters. Root rot causing fungal pathogen, Ilyonectria mors-panacis was activated on Potato Dextrose Agar (PDA) and was incubated at $25^{\circ} \mathrm{C}$. Ten day old mycelia was cut into discs of equal diameter using cork-borer and was mass produced in a commercially available vegetable juice under shaking conditions $\left(150 \mathrm{rpm}, 25^{\circ} \mathrm{C}, 15\right.$ days $)$. The pathogen was harvested and infected in artificial soil (Vermiculite, Perlite and Peat Moss - 3:1:1), in line with previously optimized protocol ${ }^{17}$. Silica nanoparticles at two different concentrations namely, $1 \mathrm{mM}$ and $2 \mathrm{mM}$ were mixed directly in soil along with the pathogen. Finally, the two-year-old homogenous ginseng seedlings were planted in pots and were cultured under controlled conditions $\left(22 \pm 2{ }^{\circ} \mathrm{C}, 12 \mathrm{~h}\right.$ light, $\left.\mathrm{RH}: 50 \pm 2 \%\right)$. Mock (HC), non-infected $1 \mathrm{mM} \mathrm{Si}(\mathrm{H}-\mathrm{Si} 1 \mathrm{mM})$, non-infected $2 \mathrm{mM} \mathrm{Si}(\mathrm{H}-\mathrm{Si} 2 \mathrm{mM})$, infected without silicon supplementation (IS), infected $1 \mathrm{mM} \mathrm{Si}$ (I-Si1mM) and infected $2 \mathrm{mM} \mathrm{Si}(\mathrm{I}-\mathrm{Si} 2 \mathrm{mM})$ were the different treatments. 
Evaluating the protective role of Si. A long term analysis for $30 \mathrm{dpi}$ was conducted to evaluate the potential of Si treatment to overcome ginseng root rot. DSI was determined by grading the ginseng seedlings based on the root phenotype at $30 \mathrm{dpi}$. DSI was calculated using the equation: $\mathrm{DSI}=[(\mathrm{X} 1 \times 1)+(\mathrm{X} 2 \times 2)+(\mathrm{X} 3 \times 3)+(\mathrm{X}$ $4 \times 4)+(\mathrm{X} 5 \times 5)+(\mathrm{X} 6 \times 6)] /(\mathrm{X} 1+\mathrm{X} 2+\mathrm{X} 3+\mathrm{X} 4+\mathrm{X} 5+\mathrm{X} 6)$, where $\mathrm{X} 1, \mathrm{X} 2, \mathrm{X} 3, \mathrm{X} 4, \mathrm{X} 5$, and X6 are the number of plants with severity scales of $1,2,3,4,5$, and 6 respectively.

Extraction and quantification of ginsenosides. Ginsenosides from the target samples were extracted and analyzed as described in a previous study ${ }^{30}$. Six freeze dried roots from all the treatments at prolonged phase II were powdered and extracted twice by refluxing with $80 \%$ methanol at $70{ }^{\circ} \mathrm{C}$ for $1 \mathrm{~h}$. The extract was filtered and in turn evaporated in a rotary evaporator at $45^{\circ} \mathrm{C}$. The resultant residue was dissolved in distilled water and was fractionated with $20 \mathrm{~mL}$ of water-saturated $n$-butanol twice. The butanol layers were mixed and evaporated to obtain crude ginsenosides. It was re-dissolved in $1 \mathrm{~mL}$ of HPLC grade methanol, filtered through $0.2 \mu \mathrm{m}$ filter and was subjected to high performance liquid chromatography (HPLC) analysis on a C18 column with water and acetonitrile as mobile phase. The major ginsenosides were quantified with a one-point curve method by employing authentic external ginsenoside standards, obtained from the Ginseng Bank, Kyung Hee University, Republic of Korea.

In-vitro analysis. The sensitivity of I. mors-panacis to silica nanoparticles was analyzed by evaluating the mycelial diameter in PDA plates. The pathogen was activated as described earlier and ten days old mycelia was cut into discs of equal diameter, then one disk was placed at the center of each plate supplemented with four concentrations of silica nanoparticles namely, $1 \mathrm{mM}, 2 \mathrm{mM}$ and $4 \mathrm{mM}$. The growth of the fungus was also analyzed on PDA without any external supplementation of silica nanoparticles and was considered as control. The growth rate of $I$. mors-panacis under various treatments was determined by using the formula: $100-[(\mathrm{dc}-\mathrm{dt}) /(\mathrm{dc}) \times 100]$, where $\mathrm{dc}=$ Mycelial diameter of control; $\mathrm{dt}=$ Mycelial diameter upon silica nanoparticlestreatment.

RNA extraction and expression profiling of target genes. Seedlings from all the treatments were sampled at early phase, intermediate phase, prolonged phase I and flash frozen in liquid nitrogen $\left(\mathrm{LN}_{2}\right)$ prior to RNA extraction, employing TRI Reagent ${ }^{\circledR}$ (Molecular Research Center, Inc, USA) with minor modifications to the standard protocol. About $100 \mathrm{mg}$ of frozen roots were ground in pre-chilled mortar in to fine powder using $\mathrm{LN}_{2} .1 \mathrm{~mL}$ of TRI Reagent was added immediately to the homogenized samples and was gently vortexed. The supernatant devoid of tissue debris were collected by centrifugation $\left(13,500 \mathrm{rpm}, 4^{\circ} \mathrm{C}\right.$ for $\left.5 \mathrm{~min}\right)$. Then, by chloroform extraction, DNA, proteins and other metabolites were precipitated, while the RNA remained in the aqueous phase. The total RNA was harvested by precipitating it from the aqueous phase using isopropanol. Exactly, $1 \mu \mathrm{g}$ of DNase treated RNA were reverse transcribed to single stranded cDNA using RevertAid Minus M-MuLV Reverse Transcriptase according to the manufacturer's instructions (Fermentas, US). Quantitative Real-time PCR was performed in a $10 \mu \mathrm{L}$ reaction volume using $\mathrm{iQ}^{\mathrm{TM}} \mathrm{SYBR}^{\circledR}$ Green Supermix in a 96 well plate. The thermal cycler conditions were, initial denaturation for $10 \mathrm{~min}$ at $95^{\circ} \mathrm{C}$, followed by 40 cycles of $95^{\circ} \mathrm{C}$ for $10 \mathrm{~s}$, annealing for $10 \mathrm{~s}$ and $72^{\circ} \mathrm{C}$ for $20 \mathrm{~s}$. The fluorescent product was perceived at the final step of each cycle. It was conducted in CFX connect Real-time PCR detection system (Bio-Rad) and comparative cycle threshold $\left(\mathrm{C}_{t}\right)$ values were documented for the target transcripts. The primer details are compiled in Table. S3. The relative quantity of the target transcript with reference to mock (HC) was determined based on $2^{-\Delta \Delta C t}$ method $^{31}$.

Extraction and profiling of methyl jasmonate. Roots from early, intermediate and prolonged I phases were flash frozen with $\mathrm{LN}_{2}$ after sampling. The frozen roots were homogenized in pre chilled mortar and $500 \mathrm{mg}$ of the fine powder was used for phytohormone extraction as described previously with few modifications ${ }^{31}$. About $5 \mathrm{~mL}$ of extraction buffer, 2-proponol/ $\mathrm{H} 2 \mathrm{O} /$ concentrated $\mathrm{HCl}(2: 1: 0.002$, vol/vol/vol) was added. After vortexing the mixture was maintained under shaking conditions at $4^{\circ} \mathrm{C}$ for $30 \mathrm{~min}$. Following it, $10 \mathrm{~mL}$ of dichloromethane was added and once again the mixture was incubated under similar shaking conditions for $30 \mathrm{~min}$. Then, upon centrifugation $(10,000 \mathrm{rpm}$ for $10 \mathrm{~min})$, two phases were formed, of which the lower phase is of interest and is evaporated at $40^{\circ} \mathrm{C}$. The samples were re-dissolved in $400 \mu \mathrm{L}$ of HPLC grade methanol and filter sterilized before LC-ESI-MS/MS analyses ${ }^{32}$.

Profiling of phytosterols by GC-MS. About $200 \mathrm{mg}$ of freeze dried powdered roots from various treatments were extracted separately for two times with $1 \mathrm{~mL}$ of $\mathrm{CHCl} 3: \mathrm{MeOH}(7: 3)$ at room temperature. $20 \mu \mathrm{g}$ of $5 \alpha$-Cholestane was employed as the internal standard. The extract upon drying in a rotary evaporator was saponified with $1.5 \mathrm{~mL}$ of $\mathrm{MeOH}$ and $\mathrm{KOH}(20 \%(\mathrm{v} / \mathrm{v}))$ at $80^{\circ} \mathrm{C}$ for $1 \mathrm{~h}$, aimed to hydrolyze the sterol esters. Following it, about $1.5 \mathrm{~mL}$ of $\mathrm{MeOH}$ and $4 \mathrm{~N} \mathrm{HCl}$ each was added and incubated at $80^{\circ} \mathrm{C}$ for $1 \mathrm{~h}$. Then, this mixture was extracted thrice with $4 \mathrm{~mL}$ of hexane. The sterols got separated to the hexane layer, which was further evaporated to dryness. The resulting powder was subjected to trimethylsilylation with pyridine and N, O-bis (trimethylsilyl) trifluoroacetamide $+1 \%$ trimethyl chlorosilane $(1: 1)$ for $90 \mathrm{~min}$ at $37^{\circ} \mathrm{C}$. Finally, GC-MS analysis was performed using a gas chromatograph (6890 A; Agilent Technologies) with a DB-5 (MS) capillary column ( $30 \mathrm{~m} \times 30.25 \mathrm{~mm}$, 0.25-mm film thickness) coupled with a mass spectrometer (HP 5973MSD). The reaction conditions were exactly similar to ${ }^{30}$. The peak area ratios of molecular ions of the endogenous sterol and that of internal standard were considered to be the actual endogenous sterol levels.

Investigation of sugar profile in the apoplastic fluid. The apoplastic fluid was extracted by following standard protocol ${ }^{10}$ with minor modifications. Panax ginseng roots were immersed in a beaker containing $1 \mathrm{~L}$ of autoclaved milliQ water and was subjected to vacuum for $5 \mathrm{~min}$. Then, the roots were cut into pieces (horizontally) and then transferred to a $15-\mathrm{mL}$ tube (with a hole punched in bottom) coupled with a $1.5 \mathrm{~mL}$ collection tube. The above mentioned set up was then inserted into a $50 \mathrm{~mL}$ template and was centrifuged at $5000 \mathrm{rpm}$ for $15 \mathrm{~min}$. The collected solution is the apoplastic fluid and was filter sterilized. Following it, the total and reducing sugars content was determined by Anthrone and DNS methods respectively. 
Quantifying target minerals. The target minerals namely, Iron (Fe), Zinc ( $\mathrm{Zn})$, Copper $(\mathrm{Cu})$ and Calcium (Ca) were quantified by Inductively Coupled Plasma with a mass spectrophotometer (ICP-MS) in the ginseng roots across all treatments and time points (Early, Intermediate, Prolonged I and Prolonged II phases). The root samples were oven-dried, ground into powder and further acid digested $\left(\mathrm{HNO}_{3}\right.$ and $\left.\mathrm{H}_{2} \mathrm{O}_{2}-1: 4\right)$. Finally, the target minerals were profiled using ICP-MS.

Statistical analysis. Standard error was calculated from three biological replications. The results were subjected to analysis of variance (ANOVA) by Duncan's multiple comparison test analysis. Mean values represented by the same letter are not significantly different from each other, while, represented by different letters are significantly different.

Received: 13 March 2019; Accepted: 8 November 2019;

Published online: 03 December 2019

\section{References}

1. Mathiyalagan, R. \& Yang, D. C. Ginseng nanoparticles: A budding tool for cancer treatment. Future Medicine (2017)

2. Kim, Y. J., Zhang, D. \& Yang, D. C. Biosynthesis and biotechnological production of ginsenosides. Biotechnol Adv. 33, 717-735 (2015).

3. Rahman, M. \& Punja, Z. K. Factors influencing development of root rot on ginseng caused by Cylindrocarpon destructans. Phytopathology. 95, 1381-1390 (2005).

4. Farh, M. E. A., Kim, Y. J., Kim, Y. J. \& Yang, D. C. Cylindrocarpon destructans/Ilyonectria radicicola-species complex: Causative agent of ginseng root-rot disease and rusty symptoms. J Ginseng Res. 42, 9-15 (2018).

5. Dodds, P. N. \& Rathjen, J. P. Plant immunity: towards an integrated view of plant-pathogen interactions. Nat. Rev. Genet. 11, 539-548 (2010).

6. Gao, Y. et al. Time-course transcriptome analysis reveals resistance genes of Panax ginseng induced by Cylindrocarpon destructans infection using RNA-seq. PloS one. 11, 0149408 (2016).

7. Rahimi, S., Kim, Y. J., Sukweenadhi, J., Zhang, D. \& Yang, D. C. PgLOX6 encoding a lipoxygenase contributes to jasmonic acid biosynthesis and ginsenoside production in Panax ginseng. J Exp Bot. 67, 6007-6019 (2016).

8. Han, J. Y., In, J. G., Kwon, Y. S. \& Choi, Y. E. Regulation of ginsenoside and phytosterol biosynthesis by RNA interferences of squalene epoxidase gene in Panax ginseng. Phytochemistry. 71, 36-46 (2010).

9. Sandor, R. et al. Plasma membrane order and fluidity are diversely triggered by elicitors of plant defence. J Exp Bot. 67, 5173-5185 (2016).

10. Wang, K., Senthil-Kumar, M., Ryu, C.M., Kang, L. \& Mysore, K.S. Phytosterols play a key role in plant innate immunity against bacterial pathogens by regulating nutrient efflux into the apoplast. Plant Physiol. 111 (2012).

11. Kim, Y. S., Balaraju, K. \& Jeon, Y. H. Biological characteristics of Bacillus amyloliquefaciens AK-0 and suppression of ginseng root rot caused by Cylindrocarpon destructans. J appl Microbiol. 122, 166-179 (2017).

12. Fauteux, F., Chain, F., Belzile, F., Menzies, J. G. \& Bélanger, R. R. The protective role of silicon in the Arabidopsis-powdery mildew pathosystem. Proc Natl Acad Sci. 103, 17554-17559 (2006).

13. Epstein, E. Silicon: its manifold roles in plants. Ann Appl Biol. 155, 155-160 (2009).

14. Van Bockhaven, J., De Vleesschauwer, D. \& Höfte, M. Towards establishing broad-spectrum disease resistance in plants: silicon leads the way. J Exp Bot. 64, 1281-1293 (2012).

15. Van Bockhaven, J. et al. Silicon induces resistance to the brown spot fungus Cochliobolus miyabeanus by preventing the pathogen from hijacking the rice ethylene pathway. New Phytol. 206, 761-773 (2015).

16. Vivancos, J., Labbé, C., Menzies, J. G. \& Bélanger, R. R. Silicon-mediated resistance of Arabidopsis against powdery mildew involves mechanisms other than the salicylic acid (SA)-dependent defence pathway. Mol Plant Pathol. 16, 572-582 (2015).

17. Suriyaprabha, R. et al. Growth and physiological responses of maize (Zea mays L.) to porous silica nanoparticles in soil. J Nanopart Res. 14, 1294 (2012).

18. Sun, D. et al. Uptake and cellular distribution, in four plant species, of fluorescently labeled mesoporous silica nanoparticles. Plant Cell Rep. 33, 1389-1402 (2014).

19. Wang, P., Lombi, E., Zhao, F. J. \& Kopittke, P. M. Nanotechnology: a new opportunity in plant sciences. Trends Plant Sci. 21, 699-712 (2016).

20. Song, A. et al. The role of silicon in enhancing resistance to bacterial blight of hydroponic-and soil-cultured rice. Sci Rep. 6, 24640 (2016).

21. Ma, J. F. \& Yamaji, N. A cooperative system of silicon transport in plants. Trends Plant Sci. 20, 435-442 (2015).

22. Ma, J. F. et al. A silicon transporter in rice. Nature. 440, 688 (2006).

23. Farh, M. E. A., Kim, Y. J., Singh, P. \& Yang, D. C. Cross interaction between Ilyonectria mors-panacis isolates infecting Korean ginseng and ginseng saponins in correlation with their pathogenicity. Phytopathology. 107, 561-569 (2017).

24. Farh, M. E. A. et al. Pathogenesis Strategies and Regulation of Ginsenosides by Two Species of Ilyonectria in Panax ginseng: Power of Speciation. J Ginseng Res. (2019)

25. Choi, J. Y. et al. Molecular characterization of MYB transcription factor genes from Panax ginseng. Russ J Plant Physl. 64, 398-409 (2017).

26. Kim, T. D., Han, J. Y., Huh, G. H. \& Choi, Y. E. Expression and functional characterization of three squalene synthase genes associated with saponin biosynthesis in Panax ginseng. Plant Cell Physiol. 52, 125-137 (2010).

27. Chen, L. Q. et al. Sugar transporters for intercellular exchange and nutrition of pathogens. Nature. 468, 527 (2010).

28. Cox, K. L. et al. TAL effector driven induction of a SWEET gene confers susceptibility to bacterial blight of cotton. Nat Commun. 8 , 15588 (2017).

29. Rahman, M. \& Punja, Z. K. Influence of iron on Cylindrocarpon root rot development on ginseng. Phytopathology. 96, 1179-1187 (2006).

30. Kim, Y.J., Lee, O.R., Oh, J.Y., Jang, M.G. \& Yang, D.C. Functional analysis of HMGR encoding genes in triterpene saponin-producing Panax ginseng Meyer. Plant Physiol. 113 (2014).

31. Schmittgen, T. D. \& Livak, K. J. Analyzing real-time PCR data by the comparative $C_{\mathrm{T}}$ method. Nat Protoc. 3, 1101 (2008).

32. Pan, X., Welti, R. \& Wang, X. Quantitative analysis of major plant hormones in crude plant extracts by high-performance liquid chromatography-mass spectrometry. Nat Protoc. 5, 986 (2010).

\section{Acknowledgements}

This research was supported by a Grant from Korea Institute of Planning \& Evaluation for Technology in Food,

Agriculture, Forestry \& Fisheries (KIPET No. 317007-3). 


\section{Author contributions}

R.A. conducted experiments, analyzed data and wrote the manuscript draft. R.A., Y.J.K. and P.M. were involved in expression profiling and interpretation. M.E.A. and D.U.Y. helped in establishment of root rot infection. Y.J.K., Ye.J.K. and R.M. designed experiments and critical revision of the manuscript. S.R. and R.V. were involved in the synthesis and characterization of silica nanoparticles. D.C.Y. and R.A. conceived idea. D.C.Y. designed experiments, interpreted data, provided overall supervision and wrote the final version of the manuscript. All the authors read and approved the manuscript.

\section{Competing interests}

The authors declare no competing interests.

\section{Additional information}

Supplementary information is available for this paper at https://doi.org/10.1038/s41598-019-54678-x.

Correspondence and requests for materials should be addressed to Y.-J.K. or D.-C.Y.

Reprints and permissions information is available at www.nature.com/reprints.

Publisher's note Springer Nature remains neutral with regard to jurisdictional claims in published maps and institutional affiliations.

(1) Open Access This article is licensed under a Creative Commons Attribution 4.0 International License, which permits use, sharing, adaptation, distribution and reproduction in any medium or format, as long as you give appropriate credit to the original author(s) and the source, provide a link to the Creative Commons license, and indicate if changes were made. The images or other third party material in this article are included in the article's Creative Commons license, unless indicated otherwise in a credit line to the material. If material is not included in the article's Creative Commons license and your intended use is not permitted by statutory regulation or exceeds the permitted use, you will need to obtain permission directly from the copyright holder. To view a copy of this license, visit http://creativecommons.org/licenses/by/4.0/.

(c) The Author(s) 2019 